\title{
Pluviosidad como Predictor de Consulta por Síndrome Febril Agudo en un Área Endémica de Dengue
}

\section{Rainfall and acute febrile syndrome in a dengue-endemic area}

Fredi A. Díaz-Quijano, Andrés L. González-Rangel, Andrea Gómez-Capacho, Reinaldo Espíndola-Gómez, Ruth A. Martínez-Vega y Luis A. Villar-Centeno

Centro de Investigaciones Epidemiológicas, Facultad de Salud, Universidad Industrial de Santander (UIS). Bucaramanga, Colombia. fre_diazq@yahoo.com, gvnkwyr@yahoo.com, andrabrun@hotmail.com, roneyesp@yahoo.com, rutharam@yahoo.com, luisangel@intercable.net.co

Recibido 13 Julio 2007/Enviado para Modificación 29 Noviembre 2007/Aceptado 12 Febrero 2008

\section{RESUMEN}

Objetivo Describir los patrones de consulta por SFA asociado al dengue y evaluar su asociación con la pluviosidad.

Métodos En dos clínicas, durante un periodo de 7 meses (de marzo a septiembre) se identificaron 318 casos de SFA indiferenciado que se incluyeron en un seguimiento clínico sistemático. El 31 \% (81/281) fueron IgM positivos para dengue. Se evaluó la pluviosidad (litros/metro cuadrado por día) previa como variable explicatoria del patrón de consultas.

Resultados Los meses con más casos de SFA fueron junio, julio y septiembre. Los casos de dengue sólo fueron significativamente más frecuentes en julio. Al evaluar los patrones de pluviosidad de las semanas anteriores, la consulta por SFA estuvo asociada con la pluviosidad promedio registrada 5 semanas antes y esta asociación fue independiente de la institución y del mes (Razón de tasas de incidencia ajustada:

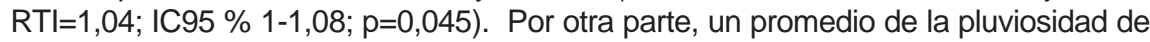
5 semanas consecutivas fue un predictor independiente de consulta por dengue, 4 semanas después (RTI=1,6; IC95 \% 1,15-2,22; $\mathrm{p}=0.006)$. Se evidenció una relación lineal entre la pluviosidad y las consultas por dengue: pluviosidad (promedio de 5 semanas) $\times 0,72($ IC95 \% 0,60-0,84; $p<0,001)=$ casos por cada 100000 habitantes en una semana (4 semanas después).

Conclusiones La pluviosidad podría predecir el patrón de consulta por dengue en una región endémica.

Palabras Clave: Dengue, cambios climáticos, intensidad de precipitación (fuente: DeCS, BIREME).

\section{ABSTRACT}

Objective Describing patterns of acute febrile syndrome (AFS) consultation related to dengue and evaluating association with rainfall. 
Methods 318 undifferentiated AFS patients were detected in two clinics during a 7month period (March to September) and then included in systematic clinical follow-up. $31 \%$ of them (81/281) were IgM positive for dengue. Rainfall (litres/square meter-day) during the previous weeks was evaluated as a variable for predicting consultation patterns.

Results June, July and September were the months in which the greatest number of AFS cases occurred. Cases of dengue were only significantly more frequent during July. When evaluating the previous weeks' rainfall patterns, consultation for AFS was seen to be associated with the average rainfall registered 5 weeks beforehand and such association was independent of the institution and/or month (incidence rate ratio IRR=1,04; $1-1.0895 \% \mathrm{Cl}, \mathrm{p}=0.045)$. On the other hand, 5 consecutive weeks' average rainfall was an independent predictor of consultation for dengue 4 weeks later on $(\mathrm{RTI}=1,6 ; 1,15-2,2295 \% \mathrm{Cl}, \mathrm{p}=0.006)$. A linear relationship was thus proposed (regarding these sentinel clinics) between consultation for dengue and rainfall: It/mtday (5 weeks' average rainfall) $\times 0,72(0,60-0,8495 \% \mathrm{Cl}, \mathrm{p}<0.001)=$ cases for each 100000 habitants per week (4 weeks later).

Conclusions: Studying rainfall could predict the pattern of consultation for dengue in endemic regions.

Key Words: Dengue, climate, rain (source: $M e S H, N L M$ ).

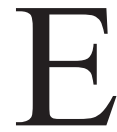

1 síndrome febril agudo (SFA) es un importante motivo de consulta en los países tropicales (1-3). Cuando clínicamente no se identifica el origen de la fiebre, los principales responsables de este síndrome son microorganismos como el dengue, el virus de la influenza, así como diversos agentes bacterianos $(2,3)$. En diversas regiones, el dengue ha sido reconocido como el principal organismo asociado a SFA de origen inaparente $(2,3)$.

Sin embargo, el estudio de esta arbovirosis, en aspectos como su presentación clínica, pronóstico y tratamiento, se ha visto limitado por la dificultad para identificar tempranamente los casos de SFA que corresponden a dengue (4). De hecho, es usual que los estudio sobre esta enfermedad se realicen en pacientes hospitalizados y la información obtenida se refiera a las fases tardías de la enfermedad cuando ya se han presentado complicaciones tales como hemorragias, extravasación plasmática o trombocitopenia profunda $(5,6)$.

Por las anteriores limitaciones, ha sido difícil caracterizar y comprender el papel del dengue en la carga de consultas por SFA de un área endémica, y reconocer su patrón de ocurrencia y sus determinantes, incluyendo factores ambientales como la pluviosidad. Esta última podría guardar relación con la densidad de vectores disponibles y, por tanto, con la intensidad de exposición al virus de los habitantes en zonas endémicas (7-9). 
En este artículo se describe la dinámica de consulta por SFA compatible con dengue en un área endémica, se estima la proporción de pacientes con SFA explicados por el dengue y se pretende evaluar el efecto de la pluviosidad sobre la incidencia de dengue y sobre la demanda asistencial por SFA. Se espera que estos resultados sean útiles para la planeación de medidas de control vectorial; la atención de estos pacientes; $y$, para la proyección de investigaciones prospectivas orientadas al estudio de los agentes causales de SFA.

\section{MATERIALES Y MÉTODOS}

Este estudio prospectivo se basó en la captación de pacientes con SFA de origen inaparente en dos instituciones centinela del área metropolitana de Bucaramanga (Colombia): Clínica Santa Teresa y Fundación Oftalmológica de Santander-Clínica Carlos Ardila Lule; las cuales llamaremos en adelante clínica A y B, respectivamente. El presente trabajo se desarrolló desde marzo hasta septiembre de 2006 (30 semanas).

En cada institución se ubicó un médico general quien permaneció en un consultorio durante 48 horas a la semana (jornada diurna) y se encargó de evaluar a los pacientes mayores de 5 años, que consultaran por SFA de menos de 96 horas de evolución. En cada clínica, se informó al personal de salud sobre el estudio y se obtuvo su colaboración para la remisión de los pacientes con estas características. Durante el periodo del estudio no se modificaron las estrategias de captación. Tanto el personal de las instituciones como los médicos encargados de la captación de pacientes desconocían las hipótesis del presente estudio.

En la consulta inicial, se evaluaron las características clínicas de cada caso y se determinó si el SFA era atribuido a una causa infecciosa evidente, como por ejemplo: amigdalitis, otitis, piodermitis, etc. En tales casos el médico instauraba el manejo correspondiente, pero éstos eran excluidos del estudio.

Por otra parte, si el paciente no tenía una causa evidente de SFA y el recuento de leucocitos era inferior a $10000 / \mu \mathrm{L}$, éste era incluido en un seguimiento clínico y de laboratorio para el estudio de su etiología. Este seguimiento incluyó la toma de una muestra en la convalecencia (después del séptimo día de enfermedad) para la realización de una prueba de ELISA para IgM específica para dengue. Se consideró como caso de dengue aquel con al menos una IgM positiva, hallazgo que en la región del estudio ha mostrado un valor predictivo positivo de $96 \%$ y especificidad de $99 \%$ (10). 
Todos los pacientes participaron voluntariamente en el estudio y dejaron constancia de ello mediante la firma de un documento de consentimiento informado, el cuál había sido revisado y aprobado por el Comité de Ética de la Facultad de Salud de la Universidad Industrial de Santander.

Adicionalmente, se recolectó información sobre la pluviosidad de la región durante el año 2006. Esta información fue aportada por el Instituto de Estudios Ambientales y Meteorológicos (IDEAM) y se basó en las observaciones en dos puntos del área metropolitana de Bucaramanga (Guatiguará y la sede del IDEAM en Bucaramanga). Las mediciones en estos dos puntos se correlacionaron significativamente $(r=0,57 ; p<0,001)$, por lo que se obtuvo un promedio diario de los dos puntos para cada semana y se expresó como litros por metro cuadrado al día.

Análisis de datos

Inicialmente se estimó la proporción de pacientes evaluados cuya etiología permaneció sin esclarecer al final del seguimiento clínico, lo cuales llamaremos SFA clínicamente indeterminados (SFACI). Luego se determinó la proporción de casos SFACI que correspondían a infecciones por dengue. Adicionalmente, se evaluó como variaba en el tiempo (cada mes) y según la institución, los valores absolutos de casos SFACI y de pacientes con dengue.

Para este último fin se tomó como unidad de análisis cada semana en cada clínica y como variable dependiente el número de pacientes que consultaron durante la misma. Se determinó si esta variable tenía una distribución normal mediante la prueba de simetría y curtosis (Skewness/Kurtosis) antes de emplear pruebas $t$ de Student y de regresión lineal. Una relación lineal se expresó como una fórmula con variable dependiente igual a intercepto más el producto de la independiente por el coeficiente de la regresión $\left(Y=\beta_{0}+\beta_{1}\right)$. Cuando el intercepto no era significativamente diferente de cero $(0)$, se obtuvo una fórmula sin esta constante.

Además, para evaluar la relación entre pluviosidad y número de consultas, también se empleó una regresión de Poisson, donde se tomó como población fuente el número de pacientes afiliados a cada clínica en la semana correspondiente. Durante el periodo del estudio esta población osciló entre 32512 y 34 887 usuarios en la clínica A y; entre 63505 y 77552 en la institución B. El número de días festivos por semana se tomó como posible variable de confusión, pues en estos días no hubo captación de pacientes. 
Posteriormente, se evaluó la asociación entre el número de pacientes incluidos en la semana y la pluviosidad promedio de la misma y de cada una de las 8 semanas anteriores. Las asociaciones encontradas fueron evaluadas en los modelos multivariados (regresión lineal y de Poisson) donde se analizó a la pluviosidad como variable explicatoria del número de pacientes. Con la regresión de Poisson se obtuvieron las razones de tasas de incidencia (RTI) como estimados de la asociación, con sus intervalos de confianza de $95 \%$ (IC95\%). El análisis de datos se realizó con el programa estadístico STATA, versión 9.2.

\section{RESULTADOS}

Se evaluaron 558 pacientes que consultaron por fiebre, de los cuales 200 presentaron evidencia de un proceso infeccioso específico durante la primera valoración y 40 más durante el seguimiento. De esta manera, 318 pacientes febriles permanecieron como SFACI al final del seguimiento ( $57 \%$ de la consulta inicial), lo que representó en promedio 5,21 pacientes por semana por institución (IC95 \% 4,63-5,79). La variable "número de casos de SFACI que consultan por semana" mostró una distribución compatible con la Normal (Prueba de simetría y curtoris: $\mathrm{p}=0,18$ ).

Comparada con la clínica $\mathrm{A}$, en la $\mathrm{B}$ consultaron más pacientes aunque esta diferencia no fue estadísticamente significativa (promedio $\mathrm{A}=4,73$; IC95 \% 3,875,$59 ;$ vs, B= 5,68; IC95 \% 4,89-6,47; p = 0,10). Sin embargo, cuando se tuvo en cuenta la población de potenciales pacientes en el modelo de Poisson, la clínica $\mathrm{B}$ mostró una tasa de captación significativamente inferior a la de la institución $\mathrm{A}$ (RTI $=0,6$; IC95 \% 0,48-0,75; $<<0,001$ ).

En relación con el mes, la tasa de captación fue mayor en las semanas de junio, julio y, posteriormente en las de septiembre (Tabla 1). Estos hallazgos fueron independientes del centro de captación y del número de días festivos y fueron consistentes con los obtenidos en los análisis de regresión (datos no mostrados).

La evaluación de la pluviosidad de la misma semana y de las anteriores a la consulta reveló que sólo la registrada 5 semanas antes tuvo una asociación estadísticamente significativa con la consulta por SFACI (RTI=1,04; IC95 \% $1,01-1,08 ; \mathrm{p}=0,004)$. Con la inclusión de esta variable en el modelo multivariado se pierde la asociación entre el número de pacientes y los meses de junio y julio (Tabla 2). En este modelo la pluviosidad mantiene su asociación con la consulta por SFACI independientemente de las demás variables consideradas 
$(p=0,045)$. La Figura 1 ilustra el número de pacientes que consultan con SFACI en las dos instituciones en cada semana y la pluviometría registrada 5 semanas antes.

Tabla 1. Variables asociadas a la tasa de captación de pacientes. Análisis de Poisson

\begin{tabular}{lccc}
\hline Variable & RTI & $($ IC 95\%) & Valor $p$ \\
\hline Mes * & & & \\
Abril & 1,14 & $(0,71-1,83)$ & 0,59 \\
Mayo & 1,18 & $(0,76-1,83)$ & 0,46 \\
Junio & 1,65 & $(1,1-2,46)$ & 0,02 \\
Julio & 1,63 & $(1,07-2,5)$ & 0,02 \\
Agosto & 1,04 & $(0,66-1,62)$ & 0,88 \\
Septiembre & 1,73 & $(1,16-2,58)$ & 0,007 \\
Clínica B & 0,56 & $(0,45-0,7)$ & $<0,001$ \\
Semana con festivo & 0,88 & $(0,66-1,16)$ & 0,36 \\
\hline
\end{tabular}

* Cada mes se comparó con marzo, el mes con menos consultas por SFA.

Tabla 2. Evaluación de la pluviosidad como un predictor de consulta por SFA en un área endémica de dengue.

\begin{tabular}{|c|c|c|c|}
\hline Variable & RTI & (IC 95\%) & Valor $\mathrm{p}$ \\
\hline Pluviosidad previa* & 1,04 & $(1-1,08)$ & 0,045 \\
\hline \multicolumn{4}{|l|}{ Mes † } \\
\hline Abril & 1,00 & $(0,61-1,64)$ & 0,99 \\
\hline Mayo & 1,05 & $(0,67-1,65)$ & 0,83 \\
\hline Junio & 1,39 & $(0,9-2,14)$ & 0,14 \\
\hline Julio & 1,34 & $(0,84-2,15)$ & 0,22 \\
\hline Agosto & 1 & $(0,64-1,56)$ & 0,99 \\
\hline Septiembre & 1,69 & $(1,13-2,52)$ & 0,01 \\
\hline Clínica B & 0,56 & $(0,45-0,7)$ & $<0,001$ \\
\hline Semana con festivo & 1,02 & $(0,74-1,4)$ & 0,92 \\
\hline
\end{tabular}

Los casos de dengue

En 37 pacientes no se realizó el estudio serológico debido a que en ellos no se pudo obtener las muestras de suero requeridas para tal fin. Por tanto, se estudiaron 281 pacientes con pruebas de IgM y de estos 81 fueron positivos (31 \%). Siguiendo un análisis similar al empleado con el total de casos de SFACI, se observó que el mes de julio estuvo asociado con un mayor número de casos de dengue (RTI: 2,53; IC95 \% 1,09-5,87; $\mathrm{p}=0,03$ ), independientemente de la institución y de los dos días festivos.

Al incluir la pluviosidad previa en este modelo, se identificaron como estadísticamente asociadas con el número de casos de dengue, la pluviosidad de las semanas precedentes 5,7 y 8 (contadas hacia atrás y considerando 0 la 
semana en la que se presentaron los casos). Las semanas 4 y 6 mostraron una tendencia a alcanzar la significación estadística (valor de p entre 0,05 y 0,1 ) en este modelo. Con la inclusión de la pluviosidad de estas semanas en el modelo, se perdió la asociación entre el mes de julio y la incidencia de dengue (Tabla 3 ).

Para obtener un indicador único de la pluviosidad, como predictor de la consulta por dengue, se obtuvo el promedio de las semanas 4 a 8 (cinco semanas) y se evaluó como predictor del número de casos que se presentarían en la semana cero. Esta variable se asoció con la consulta por dengue con un IRR=1,6 (IC95 \% 1,15-2,22; p=0.006).

Figura 1. Consulta por SFA Y Pluviosidad previs (registrada 5 semanas antes)

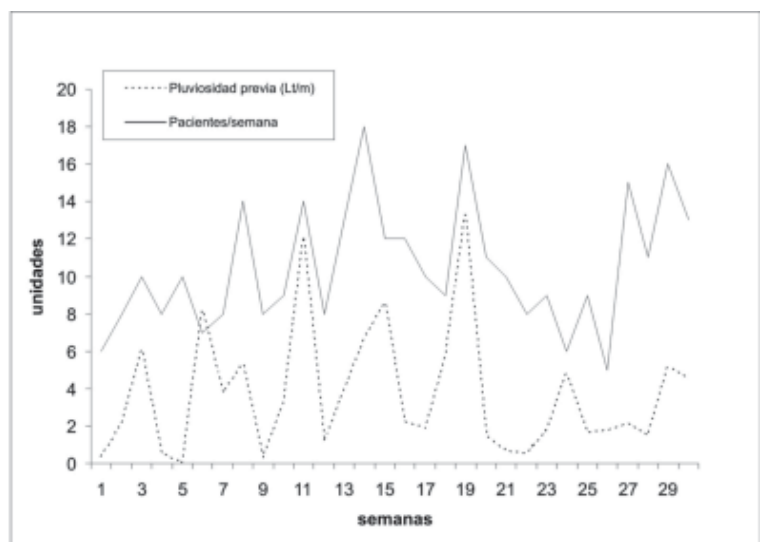

Tabla 3. Relación entre la consulta por dengue y la pluviosidad registrada previamente. Análisis de Poisson.

\begin{tabular}{|c|c|c|c|}
\hline Variable & IRR & (IC 95\%) & Valor p \\
\hline \multicolumn{4}{|l|}{ Pluviosidad previa * } \\
\hline Semana 4 & 1,08 & $(1-1,18)$ & 0,06 \\
\hline Semana 5 & 1,2 & $(1,06-1,37)$ & 0,006 \\
\hline Semana 6 & 1,12 & $(0,99-1,26)$ & 0,08 \\
\hline Semana 7 & 1,2 & $(1,06-1,37)$ & 0,005 \\
\hline Semana 8 & 1,15 & $(1,05-1,26)$ & 0,003 \\
\hline \multicolumn{4}{|l|}{ Mes $†$} \\
\hline Abril & 0,23 & $(0,04-1,35)$ & 0,10 \\
\hline Mayo & 0,34 & $(0,09-1,34)$ & 0,12 \\
\hline Junio & 0,2 & $(0,03-1,14)$ & 0,07 \\
\hline Julio & 0,26 & $(0,05-1,46)$ & 0,13 \\
\hline Agosto & 0,7 & $(0,25-1,98)$ & 0,50 \\
\hline Septiembre & 1,11 & $(0,44-2,76)$ & 0,83 \\
\hline Clínica B & 0,77 & $(0,49-1,19)$ & 0,23 \\
\hline Semana con festivo & 1,04 & $(0,55-1,94)$ & 0,91 \\
\hline
\end{tabular}


Considerando el total de potenciales pacientes (vinculados por capitación), se estimó que en promedio se presentaron 2,73 casos de dengue por semana por 100000 potenciales usuarios (IC95 \% 2,1-3,35). Esta variable (casos de dengue /100 000 usuarios-semana) mostró una distribución compatible con la normal $(p=0,66)$ y en un análisis de regresión lineal se observó una relación estadísticamente significativa entre la pluviosidad y el número de casos de dengue (fórmula sin constante ?0: número de casos por 100000 habitantes $=$ pluviometría promedio X 0,72; IC95 \% 0,60-0,84; $<<0,001$ ).

La Figura 2 ilustra el comportamiento de estas variables en el tiempo: la línea continua representa el número de consultas por dengue por cada 100000 potenciales usuarios en cada semana y, con el trazo entrecortado, se muestra la pluviosidad promedio (en litros por metro cuadrado por día) de las semanas 4 a 8 previas a la correspondiente.

Figura 2. Pluviosidad (promedio de 5 semanas) e incidencia de denque (4 semanas después)

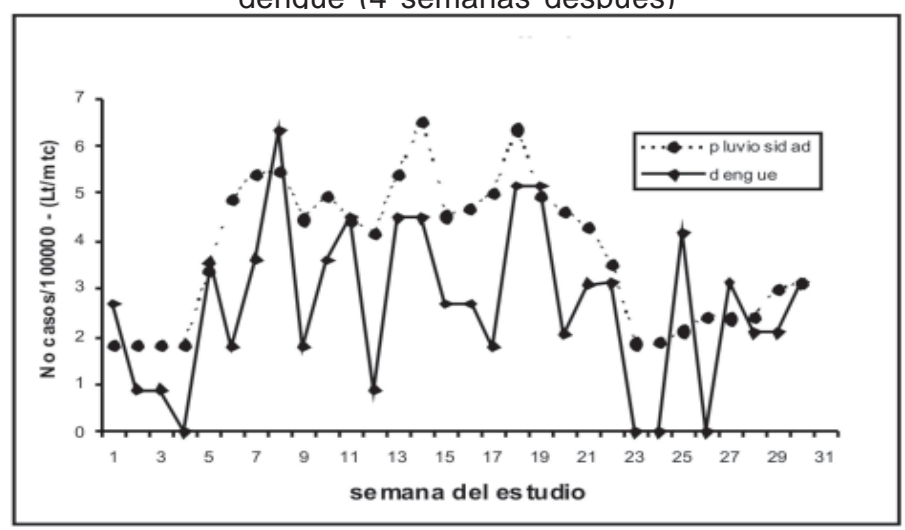

DISCUSIÓN

El presente trabajo sugiere que el SFA de origen indeterminado representa cerca del $60 \%$ de todas las consultas por fiebre a las instituciones de la región $\mathrm{y}$, de acuerdo con las pruebas de IgM realizadas, observamos que una proporción importante $(31 \%)$ de estos casos son ocasionados por dengue. Sin embargo, aún después de este estudio serológico persiste la incertidumbre diagnóstica en la mayoría de estos pacientes, lo que plantea la necesidad de buscar otros agentes causantes de SFA indiferenciado, tales como, el virus de la influenza, rubéola y las ricketsias, entre otros patógenos $(3,12)$. Esto demuestra la importancia del SFA como un reto diagnóstico y terapéutico para el clínico, así como, una prioridad de investigación. 
En el presente trabajo, se ilustra el patrón intermitente y repetitivo en los picos de aparición de pacientes con SFACI y de los casos de dengue. Como hallazgo interesante, el patrón de consulta por dengue parece explicarse en gran medida por la pluviosidad. De acuerdo con los resultados, se puede proponer que la pluviosidad promedio, de un periodo de 5 semanas, predice el número de casos de dengue que se presentarán 4 semanas después. Es plausible que las lluvias generen el ambiente propicio para el desarrollo del vector del dengue, el Aedes aegypti, quien en un periodo inferior a 4 semanas podría pasar de huevo a estado adulto en un ambiente cálido y estar disponible para infectar. Después de una picadura, se iniciaría el periodo de incubación en el huésped, lo que tomaría una semana más, antes de que se presente la consulta por $\operatorname{SFA}(13,14)$.

Con este planteamiento, es de esperar que el efecto de la pluviosidad sobre la aparición de casos febriles por dengue tarde al menos unas cinco semanas, como se evidenció en el presente estudio. Estos resultados plantean la posibilidad de establecer fórmulas para predecir la cantidad de casos de dengue que se presentarán en una región, a partir del seguimiento de los patrones de lluvias.

La fórmula obtenida en este estudio, con el análisis de regresión lineal (casos por 100000 habitantes=pluviosidad promedio x 0,72$)$, debe interpretarse con precaución pues fue obtenida en sólo dos centros centinelas. Sin embargo, ésta sugiere que puede haber una relación lineal, directamente proporcional, entre la pluviosidad y la incidencia del dengue en un área endémica.

Aunque no se realizó estudio serológico en todos los pacientes, se obtuvieron resultados de IgM en un alto porcentaje de pacientes $(88,4 \%)$. Además, es presumible que las pérdidas fueran un fenómeno aleatorio relacionado con la decisión de participación y no con las variables que se estaban evaluando. Por lo anterior, consideramos que es poco probable que estas pérdidas afectaran la asociación entre pluviosidad y casos de dengue.

Como conclusiones, el SFA de origen indeterminado es un motivo de consulta muy frecuente en nuestro medio y el dengue es una importante causa del mismo. Adicionalmente, en el periodo estudiado, el patrón cíclico de presentación del dengue pareció explicarse, al menos parcialmente, por el comportamiento de las lluvias lo que plantea la posibilidad de predecir su incidencia e instaurar medidas de control vectorial . 
Agradecimientos. Los autores agradecemos muy especialmente a la Doctora Beatriz Parra de la Universidad del Valle, por la realización de las pruebas serológicas; al personal de la Clínica Santa Teresa y de la Fundación Oftalmológica de SantanderClínica Carlos Ardila Lulle, por su colaboración en el proceso de captación de pacientes; y al Ingeniero Jaime Duarte del IDEAM, por facilitarnos la información sobre la pluviosidad de la ciudad. Financiación: Este estudio fue cofinanciado por COLCIENCIAS y la Universidad Industrial de Santander. Código de proyecto: 1102-0418205.

\section{REFERENCIAS}

1. Observatorio Departamental de Salud Pública. Indicadores de morbilidad basados en registros individuales de prestación de servicios en salud (RIPS)[Internet].Disponibleen: uww.observatorio.saludsantander.gov.co. Consultado Junio de 2007.

2. Díaz FA, Martínez RA, Villar LA. Criterios clínicos para diagnosticar el Dengue en los primeros días de enfermedad. Biomédica 2006; 26:22-30.

3. Leelarasamee A, Chupaprawan C, Chenchittikul M, Udompanthurat S. Etiologies of acute undifferentiated febrile illness in Thailand. J Med Assoc Thai 2004;87:464-72.

4. Low JG, Ooi EE, Tolfvenstam T, Leo YS, Hibberd ML, Ng LC, et al. Early Dengue Infection and Outcome Study (EDEN) - Study Design and Preliminary Findings. Ann Acad Med Singapore 2006; 35:783-7.

5. Díaz-Quijano FA, Villar-Centeno LA, Martínez-Vega RA. Complicaciones asociadas a la trombocitopenia profunda en pacientes con Dengue. Rev Méd Chile 2006; 134:167-173.

6. Balmaceda A, Hammond SN, Pérez MA, Cuadra R, Solano S, Rocha J, et al. Short Report: Assessment of the world health organization scheme for classification of dengue severity in Nicaragua. Am J Trop Med Hyg 2005; 73: 1059-62.

7. Sharma RS, Kaul SM, Sokhay J. Seasonal fluctuations of dengue fever vector, aedes aegypti diptera. Southeast Asian J Trop Med Public Health 2005; 36:186-90.

8. Thammapalo S, Chongsuwiwatwong V, McNeil D, Geater A. The climatic factors influencing the occurrence of dengue hemorrhagic. Southeast Asian J Trop Med Public Health 2005 Jan; 36:191-6.

9. Nakhapakorn K, Tripathi NK. An information value based analysis of physical and climatic factors affecting dengue fever and dengue haemorrhagic fever incidence. Int $\mathrm{J}$ Health Geogr 2005; 4:1-13.

10. Díaz-Quijano FA, Martínez-Vega RA, Ocazionez RE, Villar-Centeno LA. Evaluación de la prueba de IgM en suero agudo para el diagnóstico del dengue en un área endémica. Enferm Infecc Microbiol Clin 2006;24:90-2.

11. Diaz-Quijano FA, Villar-Centeno LA, Martinez-Vega RA. Effectiveness of early dipyrone administration on severity of dengue virus infection in a prospective cohort. Enferm Infecc Microbiol Clin 2005;23:593-7.

12. Costa L, Estétevez J, Monsalve F, Callejas D, Echevarría J. Diagnóstico etiológico de pacientes con exantemas o cuadros febriles atendidos en 1998. Estado Zulia, Venezuela. Rev Méd Chile 2004;132:1078-1084.

13. Oi FM, Anderson R. Mosquitoes In and Around the Home. University of Florida [Internet]. Disponible en: http://edis.ifas.ufl.edu/IN473\#FOOTNOTE_2. Consultado Junio de 2007.

14. Senanayake S. Dengue fever and dengue haemorrhagic fever-a diagnostic challenge. Aust Fam Physician 2006;35:609-12. 